\title{
Inequalities and asymptotic expansions related to the generalized Somos quadratic recurrence constant
}

\author{
Xue-Si Ma' and Chao-Ping Chen ${ }^{1 *}$
}

\section{"Correspondence:}

chenchaoping@sohu.com

${ }^{1}$ School of Mathematics and Informatics, Henan Polytechnic

University, Jiaozuo City, China

\section{Abstract}

In this paper, we give asymptotic expansions and inequalities related to the generalized Somos quadratic recurrence constant, using its relation with the generalized Euler constant.

MSC: $41 \mathrm{~A} 60 ; 26 \mathrm{D} 15$

Keywords: Somos' quadratic recurrence constant; Inequality; Asymptotic expansion; Generalized Euler constant

\section{Introduction}

Somos' quadratic recurrence constant is defined (see [1-3]) by

$$
\begin{aligned}
\sigma & =\sqrt{1 \sqrt{2 \sqrt{3 \cdots}}}=\prod_{n=1}^{\infty} n^{1 / 2^{n}}=\prod_{k=1}^{\infty}\left(1+\frac{1}{k}\right)^{1 / 2^{k}}=\exp \left\{\sum_{k=1}^{\infty} \frac{\ln k}{2^{k}}\right\} \\
& =1.66168794 \ldots
\end{aligned}
$$

or

$$
\sigma=\exp \left\{-\int_{0}^{1} \frac{1-x}{(2-x) \ln x} \mathrm{~d} x\right\}=\exp \left\{-\int_{0}^{1} \int_{0}^{1} \frac{x}{(2-x y) \ln (x y)} \mathrm{d} x \mathrm{~d} y\right\} .
$$

The constant $\sigma$ arises in the study of the asymptotic behavior of the sequence

$$
g_{0}=1, \quad g_{n}=n g_{n-1}^{2}, \quad n \in \mathbb{N}:=\{1,2,3, \ldots\}
$$

with the first few terms

$$
g_{0}=1, \quad g_{1}=1, \quad g_{2}=2, \quad g_{3}=12, \quad g_{4}=576, \quad g_{5}=1,658,880,
$$

This sequence behaves as follows (see [4, p. 446] and [3, 5]):

$$
\begin{aligned}
g_{n} \sim & \frac{\sigma^{2^{n}}}{n}\left(1+\frac{2}{n}-\frac{1}{n^{2}}+\frac{4}{n^{3}}-\frac{21}{n^{4}}+\frac{138}{n^{5}}-\frac{1091}{n^{6}}+\frac{10,088}{n^{7}}-\frac{106,918}{n^{8}}\right. \\
& \left.+\frac{1,279,220}{n^{9}}-\frac{17,070,418}{n^{10}}+\frac{251,560,472}{n^{11}}-\frac{4,059,954,946}{n^{12}}+\cdots\right)^{-1} .
\end{aligned}
$$

\section{Springer}


The constant $\sigma$ appears in important problems from pure and applied analysis, and it is the motivation for a large number of research papers (see, for example, [1, 6-16]).

Sondow and Hadjicostas [15] introduced and studied the generalized-Euler-constant function $\gamma(z)$, defined by

$$
\gamma(z)=\sum_{n=1}^{\infty} z^{n-1}\left(\frac{1}{n}-\ln \frac{n+1}{n}\right)
$$

where the series converges when $|z| \leq 1$. Pilehrood and Pilehrood [13] considered the function $z \gamma(z)(|z| \leq 1)$. The function $\gamma(z)$ generalizes both Euler's constant $\gamma(1)$ and the alternating Euler constant $\ln \frac{4}{\pi}=\gamma(-1)[17,18]$.

Sondow and Hadjicostas [15] defined the generalized Somos constant

$$
\sigma_{t}=\sqrt[t]{1 \sqrt[t]{2 \sqrt[t]{3 \sqrt[t]{4 \cdots}}}}=\prod_{n=1}^{\infty} n^{1 / t^{n}}=\left(\frac{t}{t-1}\right)^{1 /(t-1)} \exp \left\{-\frac{1}{t(t-1)} \gamma\left(\frac{1}{t}\right)\right\}, \quad t>1 .
$$

Coffey [19] gave the integral and series representations for $\ln \sigma_{t}$ :

$$
\ln \sigma_{t}=\int_{0}^{\infty}\left(\frac{e^{-x}}{t-1}+\frac{1}{1-t e^{x}}\right) \frac{\mathrm{d} x}{x}
$$

and

$$
\ln \sigma_{t}=\frac{1}{t-1} \sum_{k=1}^{\infty} \frac{(-1)^{k-1}}{k} \mathrm{Li}_{k}\left(\frac{1}{t}\right)=\frac{1}{t-1} \sum_{k=1}^{\infty} \frac{1}{k}\left[t \operatorname{Li}_{k}\left(\frac{1}{t}\right)-1\right]
$$

in terms of the polylogarithm function.

It is known (see [15]) that

$$
\gamma\left(\frac{1}{2}\right)=2 \ln \frac{2}{\sigma}, \quad \text { equivalently, } \quad \sigma=2 \exp \left\{-\frac{1}{2} \gamma\left(\frac{1}{2}\right)\right\} .
$$

Thus, formula (1.5) is closely related to Somos' quadratic recurrence constant $\sigma$.

Define

$$
\gamma_{n}(z)=\sum_{k=1}^{n} z^{k-1}\left(\frac{1}{k}-\ln \frac{k+1}{k}\right), \quad|z| \leq 1 .
$$

Mortici [11] proved that for $n \in \mathbb{N}$,

$$
\frac{270(n+1)}{2^{n}\left(270 n^{3}+1530 n^{2}+1065 n+6293\right)}<\gamma\left(\frac{1}{2}\right)-\gamma_{n}\left(\frac{1}{2}\right)<\frac{18}{2^{n}\left(18 n^{2}+84 n-13\right)}
$$

and

$$
\begin{aligned}
& \sum_{k=n+1}^{\infty} \frac{1}{2 k^{2} \cdot 3^{k-1}}-\frac{22,400(n+1)}{3^{n}\left(44,800 n^{4}+280,000 n^{3}+435,120 n^{2}+744,380 n-2,477,677\right)} \\
& \quad<\gamma\left(\frac{1}{3}\right)-\gamma_{n}\left(\frac{1}{3}\right)<\sum_{k=n+1}^{\infty} \frac{1}{2 k^{2} \cdot 3^{k-1}}-\frac{160}{3^{n}\left(320 n^{3}+1680 n^{2}+1428 n+3889\right)} .
\end{aligned}
$$


Lu and Song [10] improved Mortici's results and obtained the inequalities:

$$
\begin{aligned}
\frac{690 n^{2}+3524 n+145}{6\left(2^{n}\right)(n+1)^{2}\left(115 n^{2}+894 n+779\right)} & <\gamma\left(\frac{1}{2}\right)-\gamma_{n}\left(\frac{1}{2}\right) \\
& <\frac{48 n+127}{3\left(2^{n}\right)(16 n+85)(n+1)^{2}}
\end{aligned}
$$

and

$$
\begin{aligned}
& \sum_{k=n+1}^{\infty} \frac{1}{2 k^{2} \cdot 3^{k-1}}-\frac{987840 n^{2}+8444340 n+10946779}{40\left(3^{n}\right)(n+1)^{2}\left(49392 n^{3}+582741 n^{2}+1769516 n+1236167\right)} \\
& \quad<\gamma\left(\frac{1}{3}\right)-\gamma_{n}\left(\frac{1}{3}\right)<\sum_{k=n+1}^{\infty} \frac{1}{2 k^{2} \cdot 3^{k-1}}-\frac{1620 n^{2}+6995 n+1847}{40\left(3^{n}\right)\left(81 n^{2}+532 n+451\right)(n+1)^{3}}
\end{aligned}
$$

for $n \in \mathbb{N}$.

You and Chen [16] further improved inequalities (1.10)-(1.13). Recently, Chen and Han [7] gave new bounds for $\gamma(1 / 2)-\gamma_{n}(1 / 2)$ :

$$
\begin{aligned}
\frac{1}{2^{n}} & \left(\frac{1}{(n+1)^{2}}-\frac{8}{3(n+1)^{3}}+\frac{23}{2(n+1)^{4}}-\frac{332}{5(n+1)^{5}}+\frac{479}{(n+1)^{6}}-\frac{29,024}{7(n+1)^{7}}\right) \\
& <\gamma\left(\frac{1}{2}\right)-\gamma_{n}\left(\frac{1}{2}\right) \\
& <\frac{1}{2^{n}}\left(\frac{1}{(n+1)^{2}}-\frac{8}{3(n+1)^{3}}+\frac{23}{2(n+1)^{4}}-\frac{332}{5(n+1)^{5}}+\frac{479}{(n+1)^{6}}\right)
\end{aligned}
$$

for $n \in \mathbb{N}$, and presented the following asymptotic expansion:

$$
\begin{aligned}
& \gamma\left(\frac{1}{2}\right)-\gamma_{n}\left(\frac{1}{2}\right) \\
& \quad \sim \frac{1}{2^{n}(n+1)^{2}}\left\{1-\frac{8}{3(n+1)}+\frac{23}{2(n+1)^{2}}-\frac{332}{5(n+1)^{3}}+\frac{479}{(n+1)^{4}}-\cdots\right\}
\end{aligned}
$$

as $n \rightarrow \infty$. Moreover, these authors gave a formula for successively determining the coefficients in (1.15).

Chen and Han [7] pointed out that the lower bound in (1.14) is for $n \geq 24$ sharper than the one in (1.12), and the upper bound in (1.14) is for $n \geq 18$ sharper than the one in (1.12),

For any positive integer $m \geq 2$, in this paper we give the asymptotic expansion of $\gamma(1 / m)-\gamma_{n}(1 / m)$ as $n \rightarrow \infty$. Based on the result obtained, we establish the inequality for $\gamma(1 / 4)-\gamma_{n}(1 / 4)$. We also consider the asymptotic expansion for $\gamma(-1)-\gamma_{n}(-1)$.

\section{Lemmas}

Lemma 2.1 As $x \rightarrow \infty$,

$$
\frac{1}{x}-\ln \left(1+\frac{1}{x}\right)-\sum_{j=2}^{m-1} \frac{(-1)^{j}}{j} \frac{1}{x^{j}} \sim A(x)-\frac{1}{m} A(x+1),
$$


where $A(x)$ is defined by

$$
A(x)=\sum_{j=m}^{\infty} \frac{a_{j}}{x^{j}}
$$

with the coefficients $a_{j}$ given by the recurrence relation

$$
a_{j}=\frac{(-1)^{j}}{m-1}\left\{\frac{m}{j}+\sum_{k=m}^{j-1}(-1)^{k} a_{k}\left(\begin{array}{l}
j-1 \\
j-k
\end{array}\right)\right\}, \quad j \geq m
$$

Here, and throughout this paper, an empty sum is understood to be zero.

Proof Using the Maclaurin series of $\ln (1+t)$,

$$
\ln (1+t)=\sum_{j=1}^{\infty} \frac{(-1)^{j-1}}{j} t^{j}, \quad-1<t \leq 1
$$

we obtain

$$
\frac{1}{x}-\ln \left(1+\frac{1}{x}\right)-\sum_{j=2}^{m-1} \frac{(-1)^{j}}{j} \frac{1}{x^{j}}=\sum_{j=m}^{\infty} \frac{(-1)^{j}}{j} \frac{1}{x^{j}}
$$

In view of (2.4), we can let

$$
\frac{1}{x}-\ln \left(1+\frac{1}{x}\right)-\sum_{j=2}^{m-1} \frac{(-1)^{j}}{j} \frac{1}{x^{j}} \sim \sum_{j=m}^{\infty} \frac{a_{j}}{x^{j}}-\frac{1}{m} \sum_{j=m}^{\infty} \frac{a_{j}}{(x+1)^{j}},
$$

where $a_{j}$ are real numbers to be determined.

Write (2.5) as

$$
\frac{1}{x}-\ln \left(1+\frac{1}{x}\right)-\sum_{j=2}^{m-1} \frac{(-1)^{j}}{j} \frac{1}{x^{j}} \sim \sum_{j=m}^{\infty} \frac{a_{j}}{x^{j}}-\frac{1}{m} \sum_{j=m}^{\infty} \frac{a_{j}}{x^{j}}\left(1+\frac{1}{x}\right)^{-j} .
$$

Direct computation yields

$$
\begin{aligned}
\sum_{j=m}^{\infty} \frac{a_{j}}{x^{j}}\left(1+\frac{1}{x}\right)^{-j} & =\sum_{j=m}^{\infty} \frac{a_{j}}{x^{j}} \sum_{k=0}^{\infty}\left(\begin{array}{c}
-j \\
k
\end{array}\right) \frac{1}{x^{k}} \\
& =\sum_{j=m}^{\infty} \frac{a_{j}}{x^{j}} \sum_{k=0}^{\infty}(-1)^{k}\left(\begin{array}{c}
k+j-1 \\
k
\end{array}\right) \frac{1}{x^{k}} \\
& =\sum_{j=m}^{\infty} \sum_{k=m}^{j} a_{k}(-1)^{j-k}\left(\begin{array}{c}
j-1 \\
j-k
\end{array}\right) \frac{1}{x^{j}} .
\end{aligned}
$$

It follows from (2.4), (2.6), and (2.7) that

$$
\sum_{j=m}^{\infty} \frac{(-1)^{j}}{j} \frac{1}{x^{j}} \sim \sum_{j=m}^{\infty}\left\{a_{j}-\frac{1}{m} \sum_{k=m}^{j} a_{k}(-1)^{j-k}\left(\begin{array}{c}
j-1 \\
j-k
\end{array}\right)\right\} \frac{1}{x^{j}}
$$


Equating coefficients of the term $x^{-j}$ on both sides of (2.8) yields

$$
\frac{(-1)^{j}}{j}=a_{j}-\frac{1}{m} \sum_{k=m}^{j} a_{k}(-1)^{j-k}\left(\begin{array}{l}
j-1 \\
j-k
\end{array}\right), \quad j \geq m .
$$

For $j=m$, we obtain $a_{m}=\frac{(-1)^{m}}{m-1}$, and for $j \geq m+1$, we have

$$
\frac{(-1)^{j}}{j}=a_{j}-\frac{1}{m}\left[\sum_{k=m}^{j-1} a_{k}(-1)^{j-k}\left(\begin{array}{c}
j-1 \\
j-k
\end{array}\right)+a_{j}\right], \quad j \geq m+1
$$

We then obtain the recursive formula

$$
a_{m}=\frac{(-1)^{m}}{m-1}, \quad a_{j}=\frac{(-1)^{j} m}{(m-1) j}+\frac{1}{m-1} \sum_{k=m}^{j-1} a_{k}(-1)^{j-k}\left(\begin{array}{c}
j-1 \\
j-k
\end{array}\right), \quad j \geq m+1,
$$

which can be written as (2.3). The proof of Lemma 2.1 is complete.

Lemma 2.2 Let

$$
a(x)=\frac{1}{3 x^{4}}-\frac{32}{45 x^{5}} \quad \text { and } \quad b(x)=\frac{1}{3 x^{4}}-\frac{32}{45 x^{5}}+\frac{68}{27 x^{6}}
$$

Then, for $x \geq 1$,

$$
a(x)-\frac{1}{4} a(x+1)<\frac{1}{x}-\ln \left(1+\frac{1}{x}\right)-\frac{1}{2 x^{2}}+\frac{1}{3 x^{3}}<b(x)-\frac{1}{4} b(x+1) .
$$

Proof It is well known that for $-1<t \leq 1$ and $m \in \mathbb{N}$,

$$
\sum_{j=1}^{2 m} \frac{(-1)^{j-1}}{j} t^{j}<\ln (1+t)<\sum_{j=1}^{2 m-1} \frac{(-1)^{j-1}}{j} t^{j}
$$

which implies that for $x \geq 1$ and $m \geq 2$,

$$
\sum_{j=4}^{2 m+1} \frac{(-1)^{j}}{j x^{j}}<\frac{1}{x}-\ln \left(1+\frac{1}{x}\right)-\frac{1}{2 x^{2}}+\frac{1}{3 x^{3}}<\sum_{j=4}^{2 m} \frac{(-1)^{j}}{j x^{j}}
$$

Using (2.12), we find that

$$
\begin{aligned}
\frac{1}{x} & -\ln \left(1+\frac{1}{x}\right)-\frac{1}{2 x^{2}}+\frac{1}{3 x^{3}}-a(x)+\frac{1}{4} a(x+1) \\
& >\frac{1}{4 x^{4}}-\frac{1}{5 x^{5}}-a(x)+\frac{1}{4} a(x+1)=\frac{310 x^{4}+770 x^{3}+845 x^{2}+445 x+92}{180 x^{5}(x+1)^{5}}>0
\end{aligned}
$$

and

$$
\frac{1}{x}-\ln \left(1+\frac{1}{x}\right)-\frac{1}{2 x^{2}}+\frac{1}{3 x^{3}}-b(x)+\frac{1}{4} b(x+1)
$$




$$
\begin{aligned}
& <\frac{1}{4 x^{4}}-\frac{1}{5 x^{5}}+\frac{1}{6 x^{6}}-b(x)+\frac{1}{4} b(x+1) \\
& =-\frac{4380 x^{5}+14,205 x^{4}+21,530 x^{3}+17,439 x^{2}+7344 x+1270}{540 x^{6}(x+1)^{6}}<0 .
\end{aligned}
$$

The proof of Lemma 2.2 is complete.

Remark 2.1 Using the methods from [20-22] it is possible to get estimations (based on the power series expansions) of the logarithm function that can be used, for example, in the analysis of parameterized Euler-constant function, which will be an item for further work.

Lemma 2.3 As $x \rightarrow \infty$, we have

$$
\frac{1}{x}-\ln \left(1+\frac{1}{x}\right) \sim C(x)+C(x+1)
$$

where $C(x)$ is defined by

$$
C(x)=\sum_{j=2}^{\infty} \frac{c_{j}}{x^{j}}
$$

with the coefficients $c_{j}$ given by the recurrence relation

$$
c_{2}=\frac{1}{4}, \quad c_{j}=\frac{(-1)^{j}}{2 j}-\frac{1}{2} \sum_{k=2}^{j-1} c_{k}(-1)^{j-k}\left(\begin{array}{c}
j-1 \\
j-k
\end{array}\right), \quad j \geq 3 .
$$

Proof In view of (2.4), we can let

$$
\frac{1}{x}-\ln \left(1+\frac{1}{x}\right) \sim \sum_{j=2}^{\infty} \frac{c_{j}}{x^{j}}+\sum_{j=2}^{\infty} \frac{c_{j}}{(x+1)^{j}}
$$

where $c_{j}$ are real numbers to be determined. Write (2.16) as

$$
\frac{1}{x}-\ln \left(1+\frac{1}{x}\right) \sim \sum_{j=2}^{\infty} \frac{c_{j}}{x^{j}}+\sum_{j=2}^{\infty} \frac{c_{j}}{x^{j}}\left(1+\frac{1}{x}\right)^{-j}
$$

Noting that (2.7) holds, we have

$$
\sum_{j=2}^{\infty} \frac{(-1)^{j}}{j} \frac{1}{x^{j}} \sim \sum_{j=2}^{\infty}\left\{c_{j}+\sum_{k=2}^{j} c_{k}(-1)^{j-k}\left(\begin{array}{c}
j-1 \\
j-k
\end{array}\right)\right\} \frac{1}{x^{j}}
$$

Equating coefficients of the term $x^{-j}$ on both sides of (2.17) yields

$$
\frac{(-1)^{j}}{j}=c_{j}+\sum_{k=2}^{j} c_{k}(-1)^{j-k}\left(\begin{array}{l}
j-1 \\
j-k
\end{array}\right), \quad j \geq 2
$$


For $j=2$, we obtain $c_{2}=1 / 4$, and for $j \geq 3$ we have

$$
\frac{(-1)^{j}}{j}=2 c_{j}+\sum_{k=2}^{j-1} c_{k}(-1)^{j-k}\left(\begin{array}{l}
j-1 \\
j-k
\end{array}\right), \quad j \geq 3 .
$$

We then obtain the recursive formula (2.15). The proof of Lemma 2.3 is complete.

The first few coefficients $c_{j}$ are

$$
\begin{aligned}
& c_{2}=\frac{1}{4}, \quad c_{3}=\frac{1}{12}, \quad c_{4}=-\frac{1}{8}, \quad c_{5}=-\frac{1}{10}, \quad c_{6}=\frac{1}{4}, \\
& c_{7}=\frac{17}{56}, \quad c_{8}=-\frac{17}{16}, \quad c_{9}=-\frac{31}{18} .
\end{aligned}
$$

\section{Main results}

For any positive integer $m \geq 2$, Theorem 3.1 gives the asymptotic expansion of $\gamma(1 / m)-$ $\gamma_{n}(1 / m)$ as $n \rightarrow \infty$.

Theorem 3.1 For any positive integer $m \geq 2$, we have

$$
\gamma\left(\frac{1}{m}\right)-\gamma_{n}\left(\frac{1}{m}\right) \sim \sum_{k=n+1}^{\infty} \sum_{j=2}^{m-1} \frac{(-1)^{j}}{j \cdot m^{k-1}} \frac{1}{k^{j}}+\frac{A(n+1)}{m^{n}}, \quad n \rightarrow \infty
$$

where $A(x)$ is given in (2.2). Namely,

$$
\begin{aligned}
& \gamma\left(\frac{1}{m}\right)-\gamma_{n}\left(\frac{1}{m}\right) \\
& \sim \sum_{k=n+1}^{\infty} \sum_{j=2}^{m-1} \frac{(-1)^{j}}{j \cdot m^{k-1}} \frac{1}{k^{j}} \\
& \quad+\frac{(-1)^{m}}{m^{n}}\left\{\frac{1}{(m-1)(n+1)^{m}}-\frac{2 m^{2}}{(m+1)(m-1)^{2}(n+1)^{m+1}}\right. \\
& \quad+\frac{m^{2}\left(m^{2}+8 m+3\right)}{2(m+2)(m-1)^{3}(n+1)^{m+2}}-\frac{(m+1)\left(m^{3}+12 m^{2}+51 m+8\right) m^{2}}{6(m-1)^{4}(m+3)(n+1)^{m+3}} \\
& \left.\quad+\frac{m^{2}\left(m^{6}+25 m^{5}+216 m^{4}+866 m^{3}+1241 m^{2}+501 m+30\right)}{24(m-1)^{5}(m+4)(n+1)^{m+4}}-\cdots\right\} .
\end{aligned}
$$

Proof Write (2.1) as

$$
\frac{1}{k}-\ln \left(1+\frac{1}{k}\right)-\sum_{j=2}^{m-1} \frac{(-1)^{j}}{j} \frac{1}{k^{j}}=A_{N}(k)-\frac{1}{m} A_{N}(k+1)+O\left(\frac{1}{k^{N+1}}\right)
$$

where

$$
A_{N}(k)=\sum_{j=m}^{N} \frac{a_{j}}{k^{j}}
$$


with the coefficients $a_{j}$ given by the recurrence relation (2.3). From (3.3), we have

$$
\begin{gathered}
\frac{1}{m^{k-1}}\left(\frac{1}{k}-\ln \left(1+\frac{1}{k}\right)\right)-\frac{1}{m^{k-1}} \sum_{j=2}^{m-1} \frac{(-1)^{j}}{j} \frac{1}{k^{j}} \\
=\frac{A_{N}(k)}{m^{k-1}}-\frac{A_{N}(k+1)}{m^{k}}+O\left(\frac{1}{m^{k-1} k^{N+1}}\right) .
\end{gathered}
$$

Adding (3.5) from $k=n+1$ to $k=\infty$, we have

$$
\gamma\left(\frac{1}{m}\right)-\gamma_{n}\left(\frac{1}{m}\right)-\sum_{k=n+1}^{\infty} \sum_{j=2}^{m-1} \frac{(-1)^{j}}{j \cdot m^{k-1}} \frac{1}{k^{j}}=\frac{1}{m^{n}}\left\{A_{N}(n+1)+O\left(\frac{1}{(n+1)^{N+1}}\right)\right\},
$$

which can be written as (3.1). The proof of Theorem 3.1 is complete.

Remark 3.1 For $m=2$ in (3.2), we obtain (1.15). For $m=3$ in (3.2), we find

$$
\begin{aligned}
& \gamma\left(\frac{1}{3}\right)-\gamma_{n}\left(\frac{1}{3}\right) \\
& \sim \sum_{k=n+1}^{\infty} \frac{1}{2 k^{2} 3^{k-1}} \\
& \quad+\frac{1}{3^{n}(n+1)^{3}}\left\{-\frac{1}{2}+\frac{9}{8(n+1)}-\frac{81}{20(n+1)^{2}}+\frac{37}{2(n+1)^{3}}-\frac{5661}{56(n+1)^{4}}+\cdots\right\} .
\end{aligned}
$$

For $m=4$ in (3.2), we find

$$
\begin{aligned}
\gamma\left(\frac{1}{4}\right) & -\gamma_{n}\left(\frac{1}{4}\right) \\
\sim & \sum_{k=n+1}^{\infty}\left(\frac{1}{2}-\frac{1}{3 k}\right) \frac{1}{k^{2} 4^{k-1}} \\
& +\frac{1}{4^{n}(n+1)^{4}}\left\{\frac{1}{3}-\frac{32}{45(n+1)}+\frac{68}{27(n+1)^{2}}-\frac{2080}{189(n+1)^{3}}\right. \\
& \left.+\frac{9017}{162(n+1)^{4}}-\cdots\right\} .
\end{aligned}
$$

Formula (3.7) motivated us to establish Theorem 3.2.

Theorem 3.2 For $n \in \mathbb{N}$,

$$
\begin{aligned}
& \sum_{k=n+1}^{\infty}\left(\frac{1}{2}-\frac{1}{3 k}\right) \frac{1}{k^{2} 4^{k-1}}+\frac{1}{4^{n}}\left\{\frac{1}{3(n+1)^{4}}-\frac{32}{45(n+1)^{5}}\right\} \\
& \quad<\gamma\left(\frac{1}{4}\right)-\gamma_{n}\left(\frac{1}{4}\right) \\
& \quad<\sum_{k=n+1}^{\infty}\left(\frac{1}{2}-\frac{1}{3 k}\right) \frac{1}{k^{2} 4^{k-1}}+\frac{1}{4^{n}}\left\{\frac{1}{3(n+1)^{4}}-\frac{32}{45(n+1)^{5}}+\frac{68}{27(n+1)^{6}}\right\}
\end{aligned}
$$


Proof From the double inequality (2.11), we have

$$
\begin{aligned}
\frac{a(k)}{4^{k-1}}-\frac{a(k+1)}{4^{k}} & <\frac{1}{4^{k-1}}\left(\frac{1}{k}-\ln \left(1+\frac{1}{k}\right)\right)-\frac{1}{4^{k-1}}\left(\frac{1}{2 k^{2}}-\frac{1}{3 k^{3}}\right) \\
& <\frac{b(k)}{4^{k-1}}-\frac{b(k+1)}{4^{k}},
\end{aligned}
$$

where $a(x)$ and $b(x)$ are given in (2.10). Adding inequalities (3.9) from $k=n+1$ to $k=\infty$, we have

$$
\frac{a(n+1)}{4^{n}}<\sum_{k=n+1}^{\infty} \frac{1}{4^{k-1}}\left(\frac{1}{k}-\ln \left(1+\frac{1}{k}\right)\right)-\sum_{k=n+1}^{\infty} \frac{1}{4^{k-1}}\left(\frac{1}{2 k^{2}}-\frac{1}{3 k^{3}}\right)<\frac{b(n+1)}{4^{n}},
$$

which can be written as (3.8). The proof of Theorem 3.2 is complete.

Remark 3.2 Inequality (3.8) can be further refined by inserting additional terms on both sides of the inequality. For example, for $n \in \mathbb{N}$, we have

$$
\begin{aligned}
& \sum_{k=n+1}^{\infty}\left(\frac{1}{2}-\frac{1}{3 k}\right) \frac{1}{k^{2} 4^{k-1}}+\frac{1}{4^{n}}\left\{\frac{1}{3(n+1)^{4}}-\frac{32}{45(n+1)^{5}}+\frac{68}{27(n+1)^{6}}-\frac{2080}{189(n+1)^{7}}\right\} \\
& <\gamma\left(\frac{1}{4}\right)-\gamma_{n}\left(\frac{1}{4}\right) \\
& \quad<\sum_{k=n+1}^{\infty}\left(\frac{1}{2}-\frac{1}{3 k}\right) \frac{1}{k^{2} 4^{k-1}} \\
& \quad+\frac{1}{4^{n}}\left\{\frac{1}{3(n+1)^{4}}-\frac{32}{45(n+1)^{5}}+\frac{68}{27(n+1)^{6}}-\frac{2080}{189(n+1)^{7}}+\frac{9017}{162(n+1)^{8}}\right\} .
\end{aligned}
$$

Remark 3.3 Following the same method as the one used in the proof of Theorem 3.2, we can prove the following inequality:

$$
\begin{aligned}
& \sum_{k=n+1}^{\infty} \frac{1}{2 k^{2} 3^{k-1}}+\frac{1}{3^{n}}\left\{-\frac{1}{2(n+1)^{3}}+\frac{9}{8(n+1)^{4}}-\frac{81}{20(n+1)^{5}}+\frac{37}{2(n+1)^{6}}-\frac{5661}{56(n+1)^{7}}\right\} \\
& \quad<\gamma\left(\frac{1}{3}\right)-\gamma_{n}\left(\frac{1}{3}\right) \\
& \quad<\sum_{k=n+1}^{\infty} \frac{1}{2 k^{2} 3^{k-1}}+\frac{1}{3^{n}}\left\{-\frac{1}{2(n+1)^{3}}+\frac{9}{8(n+1)^{4}}-\frac{81}{20(n+1)^{5}}+\frac{37}{2(n+1)^{6}}\right\}
\end{aligned}
$$

for $n \in \mathbb{N}$. We omit the proof.

In view of (1.14), (3.11), (3.8), and (3.10), we pose the following conjecture.

Conjecture 3.1 For any positive integer $m \geq 2$, we have

$$
\begin{aligned}
\frac{(-1)^{m}}{m^{n}} \sum_{j=m}^{2 N} \frac{a_{j}}{(n+1)^{j}} & <(-1)^{m}\left\{\gamma\left(\frac{1}{m}\right)-\gamma_{n}\left(\frac{1}{m}\right)-\sum_{k=n+1}^{\infty} \sum_{j=2}^{m-1} \frac{(-1)^{j}}{j \cdot m^{k-1} k^{j}}\right\} \\
& <\frac{(-1)^{m}}{m^{n}} \sum_{j=m}^{2 N+1} \frac{a_{j}}{(n+1)^{j}},
\end{aligned}
$$


with the coefficients $a_{j}$ given in (2.3).

By using the Maple software, we find, as $n \rightarrow \infty$,

$$
\begin{aligned}
& \gamma\left(\frac{1}{2}\right)-\gamma_{n}\left(\frac{1}{2}\right) \sim \frac{1}{2^{n}(n+1)^{2}}\left(1+\frac{-\frac{8}{3}}{n+\frac{85}{16}}+\frac{-\frac{2689}{160}}{\left(n+\frac{807,797}{129,072}\right)^{3}}+\cdots\right), \\
& \gamma\left(\frac{1}{3}\right)-\gamma_{n}\left(\frac{1}{3}\right) \\
& \quad \sim \sum_{k=n+1}^{\infty} \frac{1}{2 k^{2} 3^{k-1}}+\frac{1}{3^{n}(n+1)^{3}}\left(-\frac{1}{2}+\frac{\frac{9}{8}}{n+\frac{23}{5}}+\frac{\frac{98}{25}}{\left(n+\frac{140,843}{27,440}\right)^{3}}+\cdots\right)
\end{aligned}
$$

and

$$
\begin{aligned}
\gamma\left(\frac{1}{4}\right)-\gamma_{n}\left(\frac{1}{4}\right) \sim & \sum_{k=n+1}^{\infty}\left(\frac{1}{2}-\frac{1}{3 k}\right) \frac{1}{k^{2} 4^{k-1}} \\
& +\frac{1}{4^{n}(n+1)^{4}}\left\{\frac{1}{3}+\frac{-\frac{32}{45}}{n+\frac{109}{24}}+\frac{-\frac{2365}{1134}}{\left(n+\frac{825,361}{170,280}\right)^{3}}+\cdots\right\} .
\end{aligned}
$$

From a computational viewpoint, formulas (3.13), (3.14), and (3.15) improve formulas (1.15), (3.6), and (3.7), respectively.

For any positive integer $m \geq 2$, we here provide a pair of recurrence relations for determining the constants $p_{\ell} \equiv p_{\ell}(m)$ and $q_{\ell} \equiv q_{\ell}(m)$ (see Remark 3.4) such that

$$
\begin{aligned}
& \gamma\left(\frac{1}{m}\right)-\gamma_{n}\left(\frac{1}{m}\right) \\
& \quad \sim \sum_{k=n+1}^{\infty} \sum_{j=2}^{m-1} \frac{(-1)^{j}}{j \cdot m^{k-1}} \frac{1}{k^{j}}+\frac{1}{m^{n}(n+1)^{m}}\left(a_{m}+\sum_{\ell=1}^{\infty} \frac{p_{\ell}}{\left(n+q_{\ell}\right)^{2 \ell-1}}\right)
\end{aligned}
$$

as $n \rightarrow \infty$. This develops formulas (3.13), (3.14), and (3.15) to produce a general result given by Theorem 3.3 .

Theorem 3.3 For any positive integer $m \geq 2$, we have

$$
\gamma\left(\frac{1}{m}\right)-\gamma_{n-1}\left(\frac{1}{m}\right) \sim \sum_{k=n}^{\infty} \sum_{j=2}^{m-1} \frac{(-1)^{j}}{j \cdot m^{k-1}} \frac{1}{k^{j}}+\frac{1}{m^{n-1} n^{m}}\left(a_{m}+\sum_{\ell=1}^{\infty} \frac{\lambda_{\ell}}{\left(n+\mu_{\ell}\right)^{2 \ell-1}}\right)
$$

as $n \rightarrow \infty$, where $\lambda_{\ell} \equiv \lambda_{\ell}(m)$ and $\mu_{\ell} \equiv \mu_{\ell}(m)$ are given by a pair of recurrence relations

$$
\lambda_{\ell}=a_{m+2 \ell-1}-\sum_{k=1}^{\ell-1} \lambda_{k} \mu_{k}^{2 \ell-2 k}\left(\begin{array}{c}
2 \ell-2 \\
2 \ell-2 k
\end{array}\right), \quad \ell \geq 2,
$$

and

$$
\mu_{\ell}=-\frac{1}{(2 \ell-1) \lambda_{\ell}}\left\{a_{m+2 \ell}+\sum_{k=1}^{\ell-1} \lambda_{k} \mu_{k}^{2 \ell-2 k+1}\left(\begin{array}{c}
2 \ell-1 \\
2 \ell-2 k+1
\end{array}\right)\right\}, \quad \ell \geq 2,
$$


with

$$
\lambda_{1}=a_{m+1}=\frac{(-1)^{m+1} 2 m^{2}}{(m+1)(m-1)^{2}} \quad \text { and } \quad \mu_{1}=-\frac{a_{m+2}}{\lambda_{1}}=\frac{(m+1)\left(m^{2}+8 m+3\right)}{4(m+2)(m-1)} .
$$

Here $a_{j}$ are given in (2.3).

Proof In view of (3.13), (3.14), and (3.15), we let

$$
\gamma\left(\frac{1}{m}\right)-\gamma_{n-1}\left(\frac{1}{m}\right) \sim \sum_{k=n}^{\infty} \sum_{j=2}^{m-1} \frac{(-1)^{j}}{j \cdot m^{k-1}} \frac{1}{k^{j}}+\frac{1}{m^{n-1} n^{m}}\left(a_{m}+\sum_{\ell=1}^{\infty} \frac{\lambda_{\ell}}{\left(n+\mu_{\ell}\right)^{2 \ell-1}}\right),
$$

where $\lambda_{\ell}$ and $\mu_{\ell}$ are real numbers to be determined. This can be written as

$$
\begin{aligned}
& m^{n-1} n^{m}\left\{\gamma\left(\frac{1}{m}\right)-\gamma_{n-1}\left(\frac{1}{m}\right)-\sum_{k=n}^{\infty} \sum_{j=2}^{m-1} \frac{(-1)^{j}}{j \cdot m^{k-1}} \frac{1}{k^{j}}\right\} \\
& \sim a_{m}+\sum_{j=1}^{\infty} \frac{\lambda_{j}}{n^{2 j-1}}\left(1+\frac{\mu_{j}}{n}\right)^{-2 j+1} .
\end{aligned}
$$

Direct computation yields

$$
\begin{aligned}
\sum_{j=1}^{\infty} \frac{\lambda_{j}}{n^{2 j-1}}\left(1+\frac{\mu_{j}}{n}\right)^{-2 j+1} & =\sum_{j=1}^{\infty} \frac{\lambda_{j}}{n^{2 j-1}} \sum_{k=0}^{\infty}\left(\begin{array}{c}
-2 j+1 \\
k
\end{array}\right) \frac{\mu_{j}^{k}}{n^{k}} \\
& =\sum_{j=1}^{\infty} \frac{\lambda_{j}}{n^{2 j-1}} \sum_{k=0}^{\infty}(-1)^{k}\left(\begin{array}{c}
k+2 j-2 \\
k
\end{array}\right) \frac{\mu_{j}^{k}}{n^{k}} \\
& =\sum_{j=1}^{\infty} \sum_{k=0}^{j-1} \lambda_{k+1} \mu_{k+1}^{j-k-1}(-1)^{j-k-1}\left(\begin{array}{c}
j+k-1 \\
j-k-1
\end{array}\right) \frac{1}{n^{j+k}},
\end{aligned}
$$

which can be written as

$$
\sum_{j=1}^{\infty} \frac{\lambda_{j}}{n^{2 j-1}}\left(1+\frac{\mu_{j}}{n}\right)^{-2 j+1} \sim \sum_{j=1}^{\infty}\left\{\sum_{k=1}^{\lfloor+2\rfloor} \lambda_{k} \mu_{k}^{j-2 k+1}(-1)^{j-1}\left(\begin{array}{c}
j-1 \\
j-2 k+1
\end{array}\right)\right\} \frac{1}{n^{j}} .
$$

Substituting (3.21) into (3.20) we have

$$
\begin{aligned}
& m^{n-1} n^{m}\left\{\gamma\left(\frac{1}{m}\right)-\gamma_{n-1}\left(\frac{1}{m}\right)-\sum_{k=n}^{\infty} \sum_{j=2}^{m-1} \frac{(-1)^{j}}{j \cdot m^{k-1}} \frac{1}{k^{j}}\right\} \\
& \sim a_{m}+\sum_{j=1}^{\infty}\left\{\sum_{k=1}^{\left\lfloor\frac{j+2}{2}\right\rfloor} \lambda_{k} \mu_{k}^{j-2 k+1}(-1)^{j-1}\left(\begin{array}{c}
j-1 \\
j-2 k+1
\end{array}\right)\right\} \frac{1}{n^{j}} .
\end{aligned}
$$

On the other hand, it follows from (3.1) that

$$
m^{n-1} n^{m}\left\{\gamma\left(\frac{1}{m}\right)-\gamma_{n-1}\left(\frac{1}{m}\right)-\sum_{k=n}^{\infty} \sum_{j=2}^{m-1} \frac{(-1)^{j}}{j \cdot m^{k-1}} \frac{1}{k^{j}}\right\} \sim \sum_{j=0}^{\infty} \frac{a_{m+j}}{n^{j}} .
$$


Equating coefficients of the term $n^{-j}$ on the right-hand sides of (3.22) and (3.23), we obtain

$$
a_{m+j}=\sum_{k=1}^{\left\lfloor\frac{j+2}{2}\right\rfloor} \lambda_{k} \mu_{k}^{j-2 k+1}(-1)^{j-1}\left(\begin{array}{c}
j-1 \\
j-2 k+1
\end{array}\right), \quad j \in \mathbb{N} .
$$

Setting $j=2 \ell-1$ and $j=2 \ell$ in (3.24), respectively, yields

$$
a_{m+2 \ell-1}=\sum_{k=1}^{\ell} \lambda_{k} \mu_{k}^{2 \ell-2 k}\left(\begin{array}{c}
2 \ell-2 \\
2 \ell-2 k
\end{array}\right)
$$

and

$$
\begin{aligned}
a_{m+2 \ell} & =-\sum_{k=1}^{\ell+1} \lambda_{k} \mu_{k}^{2 \ell-2 k+1}\left(\begin{array}{c}
2 \ell-1 \\
2 \ell-2 k+1
\end{array}\right) \\
& =-\sum_{k=1}^{\ell} \lambda_{k} \mu_{k}^{2 \ell-2 k+1}\left(\begin{array}{c}
2 \ell-1 \\
2 \ell-2 k+1
\end{array}\right)-\lambda_{\ell+1} \mu_{\ell+1}^{-1}\left(\begin{array}{c}
2 \ell-1 \\
-1
\end{array}\right) \\
& =-\sum_{k=1}^{\ell} \lambda_{k} \mu_{k}^{2 \ell-2 k+1}\left(\begin{array}{c}
2 \ell-1 \\
2 \ell-2 k+1
\end{array}\right) .
\end{aligned}
$$

For $\ell=1$, from (3.25) and (3.26) we obtain

$$
\lambda_{1}=a_{m+1}=\frac{(-1)^{m+1} 2 m^{2}}{(m+1)(m-1)^{2}} \quad \text { and } \quad \mu_{1}=-\frac{a_{m+2}}{\lambda_{1}}=\frac{(m+1)\left(m^{2}+8 m+3\right)}{4(m+2)(m-1)}
$$

and for $\ell \geq 2$ we have

$$
a_{m+2 \ell-1}=\sum_{k=1}^{\ell-1} \lambda_{k} \mu_{k}^{2 \ell-2 k}\left(\begin{array}{c}
2 \ell-2 \\
2 \ell-2 k
\end{array}\right)+\lambda_{\ell}
$$

and

$$
a_{m+2 \ell}=-\sum_{k=1}^{\ell-1} \lambda_{k} \mu_{k}^{2 \ell-2 k+1}\left(\begin{array}{c}
2 \ell-1 \\
2 \ell-2 k+1
\end{array}\right)-(2 \ell-1) \lambda_{\ell} \mu_{\ell} .
$$

We then obtain the recurrence relations (3.18) and (3.19). The proof of Theorem 3.3 is complete.

Here we give explicit numerical values of some first terms of $\lambda_{\ell}$ and $\mu_{\ell}$ by using formulas (3.18) and (3.19). This shows how easily we can determine the constants $a_{\ell}$ and $b_{\ell}$ in (3.17).

$$
\begin{aligned}
& \lambda_{1}=a_{m+1}=\frac{(-1)^{m+1} 2 m^{2}}{(m+1)(m-1)^{2}}, \\
& \mu_{1}=-\frac{a_{m+2}}{\lambda_{1}}=\frac{(m+1)\left(m^{2}+8 m+3\right)}{4(m+2)(m-1)},
\end{aligned}
$$




$$
\begin{aligned}
& \lambda_{2}=a_{m+3}-\lambda_{1} \mu_{1}^{2} \\
& =\frac{(-1)^{m+1} m^{2}(m+1)\left(m^{3}+12 m^{2}+51 m+8\right)}{6(m-1)^{4}(m+3)} \\
& -\frac{(-1)^{m+1} 2 m^{2}}{(m+1)(m-1)^{2}}\left(\frac{(m+1)\left(m^{2}+8 m+3\right)}{4(m+2)(m-1)}\right)^{2} \\
& =(-1)^{m+1} \frac{m^{2}(m+1)\left(m^{5}+7 m^{4}+58 m^{3}+266 m^{2}+485 m+47\right)}{24(m-1)^{4}(m+3)(m+2)^{2}} \text {, } \\
& \mu_{2}=-\frac{a_{m+4}+\lambda_{1} \mu_{1}^{3}}{3 \lambda_{2}} \\
& =-\frac{\frac{(-1)^{m} m^{2}\left(m^{6}+25 m^{5}+216 m^{4}+866 m^{3}+1241 m^{2}+501 m+30\right)}{24(m-1)^{5}(m+4)}+\lambda_{1} \mu_{1}^{3}}{3 \lambda_{2}} \\
& =\left(( m + 3 ) \left(m^{9}+34 m^{8}+450 m^{7}+3634 m^{6}+17,584 m^{5}+48,642 m^{4}+71,302 m^{3}\right.\right. \\
& \left.\left.+50,926 m^{2}+14,151 m+636\right)\right) /\left(1 2 ( m + 2 ) ( m + 4 ) \left(m^{5}+7 m^{4}+58 m^{3}+266 m^{2}\right.\right. \\
& \left.+485 m+47)\left(m^{2}-1\right)\right) \text {. }
\end{aligned}
$$

Remark 3.4 The constants $p_{\ell}$ and $q_{\ell}$ in (3.16) are given by

$$
p_{\ell}:=\lambda_{\ell} \quad \text { and } \quad q_{\ell}:=1+\mu_{\ell}
$$

Setting $m=2,3$, and 4 in (3.16), respectively, yields (3.13), (3.14), and (3.15).

Noting that $\ln \frac{4}{\pi}=\gamma(-1)$ holds, Theorem 3.4 presents the asymptotic expansion for $\ln \frac{4}{\pi}$.

Theorem 3.4 As $n \rightarrow \infty$, we have

$$
\gamma(-1)-\gamma_{n}(-1) \sim(-1)^{n} C(n+1),
$$

where $C(x)$ is given in (2.14). Namely,

$$
\begin{aligned}
& \gamma(-1)-\gamma_{n}(-1) \\
& \sim(-1)^{n}\left\{\frac{1}{4(n+1)^{2}}+\frac{1}{12(n+1)^{3}}-\frac{1}{8(n+1)^{4}}-\frac{1}{10(n+1)^{5}}+\cdots\right\}
\end{aligned}
$$

Proof Write (2.13) as

$$
\frac{1}{k}-\ln \left(1+\frac{1}{k}\right)=C_{N}(k)+C_{N}(k+1)+O\left(\frac{1}{k^{N+1}}\right)
$$

where

$$
C_{N}(x)=\sum_{j=2}^{N} \frac{c_{j}}{x^{j}}
$$

with the coefficients $c_{j}$ given by the recurrence relation (2.15).

From (3.29), we have

$$
(-1)^{k-1}\left(\frac{1}{k}-\ln \left(1+\frac{1}{k}\right)\right)=(-1)^{k-1} C_{N}(k)-(-1)^{k} C_{N}(k+1)+O\left(\frac{(-1)^{k-1}}{k^{N+1}}\right) .
$$


Adding (3.31) from $k=n+1$ to $k=\infty$, we have

$$
\begin{aligned}
\gamma(-1)-\gamma_{n}(-1) & =\sum_{k=n+1}^{\infty}(-1)^{k-1}\left(\frac{1}{k}-\ln \left(1+\frac{1}{k}\right)\right) \\
& =(-1)^{n} C_{N}(n+1)+O\left(\frac{1}{(n+1)^{N+1}}\right),
\end{aligned}
$$

which can be written as (3.27). The proof of Theorem 3.4 is complete.

Remark 3.5 We see from (3.28) that the alternating Euler constant $\ln \frac{4}{\pi}$ has the following expansion:

$$
\begin{aligned}
\ln \frac{4}{\pi} \sim & \sum_{k=1}^{n}(-1)^{k-1}\left(\frac{1}{k}-\ln \frac{k+1}{k}\right) \\
& +(-1)^{n}\left\{\frac{1}{4(n+1)^{2}}+\frac{1}{12(n+1)^{3}}-\frac{1}{8(n+1)^{4}}-\frac{1}{10(n+1)^{5}}+\cdots\right\}
\end{aligned}
$$

\section{Conclusions}

In this paper, we give asymptotic expansions related to the generalized Somos quadratic recurrence constant (Theorems 3.1 and 3.3). We present the inequalities for $\gamma\left(\frac{1}{4}\right)-\gamma_{n}\left(\frac{1}{4}\right)$ and $\gamma\left(\frac{1}{3}\right)-\gamma_{n}\left(\frac{1}{3}\right)$ (see (3.8), (3.10), and (3.11)). The expansion of the alternating Euler constant $\ln \frac{4}{\pi}$ is also obtained (see (3.33)).

\section{Acknowledgements}

We thank the editor and referees for their careful reading and valuable suggestions to make the article reader friendly.

\section{Funding}

Not applicable

\section{Competing interests}

The authors declare that they have no competing interests.

\section{Authors' contributions}

Both authors contributed equally to this work. Both authors read and approved the final manuscript.

\section{Publisher's Note}

Springer Nature remains neutral with regard to jurisdictional claims in published maps and institutional affiliations.

Received: 10 January 2018 Accepted: 19 March 2018 Published online: 27 June 2018

\section{References}

1. Guillera, J., Sondow, J.: Double integrals and infinite products for some classical constants via analytic continuations of Lerch's transcendent. Ramanujan J. 16, 247-270 (2008)

2. Ramanujan, S.: In: Hardy, G.H., Aiyar, P.V.S., Wilson, B.M. (eds.) Collected Papers of Srinivasa Ramanujan. Am. Math. Soc., Providence (2000)

3. Sloane, N.J.A.: Sequences A116603. In: The On-Line Encyclopedia of Integer Sequences. http://oeis.org/A116603

4. Finch, S.R.: Mathematical Constants. Cambridge University Press, Cambridge (2003)

5. Weisstein, E.W.: Somos's quadratic recurrence constant. In: MathWorld—A Wolfram Web Resource. Published electronically at http://mathworld.wolfram.com/SomossQuadraticRecurrenceConstant.html

6. Chen, C.P.: New asymptotic expansions related to Somos' quadratic recurrence constant. C. R. Acad. Sci. Paris, Ser. I 351, 9-12 (2013)

7. Chen, C.P., Han, X.F.: On Somos' quadratic recurrence constant. J. Number Theory 166, 31-40 (2016)

8. Hirschhorn, M.D.: A note on Somos' quadratic recurrence constant. J. Number Theory 131, 2061-2063 (2011)

9. Lampret, V.: Approximation of Sondow's generalized-Euler-constant function on the interval $[-1,1]$. Ann. Univ. Ferrara $56,65-76(2010)$

10. Lu, D., Song, Z.: Some new continued fraction estimates of the Somos' quadratic recurrence constant. J. Number Theory $155,36-45(2015)$ 
11. Mortici, C.: Estimating the Somos' quadratic recurrence constant. J. Number Theory 130, 2650-2657 (2010)

12. Nemes, G.: On the coefficients of an asymptotic expansion related to Somos' quadratic recurrence constant. Appl. Anal. Discrete Math. 5, 60-66 (2011)

13. Pilehrood, K.H., Pilehrood, T.H.: Arithmetical properties of some series with logarithmic coefficients. Math. Z. 255, 117-131 (2007)

14. Pilehrood, K.H., Pilehrood, T.H.: Vacca-type series for values of the generalized Euler constant function and its derivative. J. Integer Seq. 13, Article ID 10.7.3 (2010)

15. Sondow, J., Hadjicostas, P.: The generalized-Euler-constant function $\gamma(z)$ and a generalization of Somos's quadratic recurrence constant. J. Math. Anal. Appl. 332, 292-314 (2007)

16. You, X., Chen, D.R.: Improved continued fraction sequence convergent to the Somos' quadratic recurrence constant. J. Math. Anal. Appl. 436, 513-520 (2016)

17. Sondow, J.: Double integrals for Euler's constant and $\ln (4 / \pi)$ and an analog of Hadjicostas's formula. Am. Math. Mon. $112,61-65(2005)$

18. Sondow, J.: New Vacca-type rational series for Euler's constant and its "alternating" analog $\ln (4 / \pi)$. In: Additive Number Theory, pp. 331-340. Springer, New York (2010)

19. Coffey, M.W.: Integral representations of functions and Addison-type series for mathematical constants. J. Number Theory 157, 79-98 (2015)

20. Lutovac, T., Malesevic, B., Rasajski, M.: A new method for proving some inequalities related to several special functions (2018). arXiv:1802.02082

21. Malesevic, B., Lutovac, T., Rasajski, M., Mortici, C.: Extensions of the natural approach to refinements and generalizations of some trigonometric inequalities. Adv. Differ. Equ. 2018, 90 (2018)

22. Malesevic, B., Rasajski, M., Lutovac, T.: Refinements and generalizations of some inequalities of Shafer-Fink's type for the inverse sine function. J. Inequal. Appl. 2017, 275 (2017)

\section{Submit your manuscript to a SpringerOpen ${ }^{\circ}$ journal and benefit from:}

- Convenient online submission

- Rigorous peer review

- Open access: articles freely available online

- High visibility within the field

- Retaining the copyright to your article

Submit your next manuscript at $\gg$ springeropen.com 